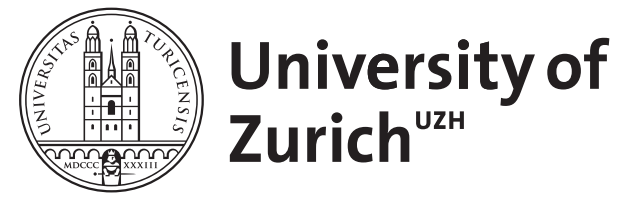

\title{
Video-assisted sacral nerve stimulation
}

\author{
Hetzer, F H ; Hahnloser, D ; Clavien, P A ; Demartines, N
}

\begin{abstract}
Background: Sacral nerve simulation (SNS) is an accepted therapy for patients with urinary or bowel dysfunction. However, infection rates are as high as $20 \%$ and can result in removal of the expensive device. We present a new video-assisted technique minimizing the risk of infection. Methods: Between April and July 2005, six consecutive women of median age 68 years (range, 60-74), with faecal incontinence (4 patients) and idiopathic constipation (2 patients) underwent video-assisted electrode implantation for SNS. The motor response of the pelvic floor during percutaneous nerve evaluation and implantation of the permanent lead was monitored by a video optic (same as that normally used for laparoscopic or endoscopic procedures) placed between the legs of the patients. The video optic and the perianal area were completely covered with drapes, separating them from the operating field. Results: All but one screening was successful, and no wound infections at the electrode or at the pocket of the stimulator were noted (mean postoperative follow-up, 8 weeks). Conclusions: With the use of a video optic, the anus and the implantation site can be completely separated and contamination during the operation becomes unlikely. Furthermore, the response of the pelvic floor to the stimulation is better visualized. We routinely recommend the use of video equipment for SNS electrode implantation
\end{abstract}

DOI: https://doi.org/10.1007/s10151-006-0264-3

Posted at the Zurich Open Repository and Archive, University of Zurich ZORA URL: https://doi.org/10.5167/uzh-155917

Journal Article

Published Version

Originally published at:

Hetzer, F H; Hahnloser, D; Clavien, P A; Demartines, N (2006). Video-assisted sacral nerve stimulation. Techniques in Coloproctology, 10(2):121-124.

DOI: https://doi.org/10.1007/s10151-006-0264-3 


\section{F.H. Hetzer • D. Hahnloser • P.A. Clavien • N. Demartines Video-assisted sacral nerve stimulation}

Received: 20 September 2005 / Accepted: 3 October 2005 / Published online: 19 June 2006

\begin{abstract}
Background Sacral nerve simulation (SNS) is an accepted therapy for patients with urinary or bowel dysfunction. However, infection rates are as high as $20 \%$ and can result in removal of the expensive device. We present a new video-assisted technique minimizing the risk of infection. Methods Between April and July 2005, six consecutive women of median age 68 years (range, 60-74), with faecal incontinence (4 patients) and idiopathic constipation (2 patients) underwent video-assisted electrode implantation for SNS. The motor response of the pelvic floor during percutaneous nerve evaluation and implantation of the permanent lead was monitored by a video optic (same as that normally used for laparoscopic or endoscopic procedures) placed between the legs of the patients. The video optic and the perianal area were completely covered with drapes, separating them from the operating field.
\end{abstract}

F.H. Hetzer $(\varangle) \cdot$ D. Hahnloser • P.A. Clavien • N. Demartines Department of Visceral and Transplantation Surgery

University Hospital

Zurich, Switzerland

E-mail: franc.hetzer@usz.ch
Results All but one screening was successful, and no wound infections at the electrode or at the pocket of the stimulator were noted (mean postoperative follow-up, 8 weeks). Conclusions With the use of a video optic, the anus and the implantation site can be completely separated and contamination during the operation becomes unlikely. Furthermore, the response of the pelvic floor to the stimulation is better visualized. We routinely recommend the use of video equipment for SNS electrode implantation.

Key words Faecal incontinence $\cdot$ Sacral nerve stimulation • Minimal invasive sacral neuromodulation • Video-assisted sacral nerve stimulation

\section{Introduction}

Since the first description of sacral nerve stimulation (SNS) by Pescatori et al. in 1982 [1] and then by Tanagho et al. [2] in 1989, SNS is becoming an accepted therapy for patients with urinary or bowel dysfunction [3-7]. In the last few years the operative technique has changed: the procedure has become minimally invasive [6] and the complex three-stage intervention was successfully altered to a two-stage implantation $[8,9]$. All these improvements have increased the success rate and have allowed clinicians to perform the procedure under local anaesthesia and in an outpatient setting [10].

The procedure's Achilles' heel, however, is the high infection rate. Infection can occur early during the screening phase or later after permanent implantation of the stimulator. Infected screening leads require removal and interruption of the testing phase; this occurs in up to $20 \%$ of patients [11]. Wound infection after implantation of the neurostimulator is treated similarly, however the loss of the stimulator dramatically increases costs. In our experience of 36 permanent implantations over a four-year peri- 
od (unpublished data), the stimulator had to be removed in one case $(2.8 \%)$, similar to the $4 \%$ removal rate in another report [12].

This report demonstrates a new video-assisted technique to avoid infection.

\section{Patients and methods}

From April to July 2005, SNS was tested in 6 women with a median age of 68 years (range, 60-74); 4 patients had faecal incontinence while 2 had idiopathic constipation. The study was conducted in accordance with the Declaration of Helsinki. After patients gave written informed consent to participate the study, the two-stage procedures were performed in an outpatient setting $(<12$ hours). All patients received a single dose of antibiotics preoperatively (1000 $\mathrm{mg}$ cefazolin and $500 \mathrm{mg}$ metronidazole intravenously).

\section{Surgical technique}

For percutaneous nerve evaluation and implantation of the permanent electrode (model 3889, Medtronic, Minneapolis, USA), patients were prone positioned with slightly bended hips and knees (Fig. 1). Patient's feet have to be free in order to observe the flexion of the great toe as response to stimulation of the third sacral root. Lateral fluoroscopy of the sacrum is essential for introducing and correctly placing the tined lead. Additionally, we placed a video optic (A9394A, 0 degree optic, light cable WAO03210A and a support A00180A, Olympus, Zurich Switzerland) between the legs in order to visualize the contraction of the pelvic floor. The monitor was placed at the patients' feet.

After disinfection of the sacral area (cranially up to the iliac crest, laterally $15-20 \mathrm{~cm}$ from the anal cleft on each side and cau-



Fig. 1 Patient in prone position with slightly bended hips and knees. Feet have to be free to permit clinicians to observe flexion of the great toe. Lateral fluoroscopy of the sacrum is essential for introducing and correctly placing the tined lead. A video optic is placed between the legs to visualize the contraction of the pelvic floor. The corresponding monitor is placed at the feet of the patient dally to the tip of the coccyx), a sterile rectangular coverage was placed. The fluoroscope, the video optic, and the perianal area were completely covered. The instrumental nurse stood on the left side of the patient's leg, and the right-handed surgeon stood on the right side.

After local anaesthesia, the sacral S3 foramina on both sides were evaluated for stimulation by introducing the foramen needle and test electrode. Additionally to the patient's sensations, the pelvic contraction was observed on the monitor. The tined lead was then implanted on the side were the lowest motoric threshold resulted and no disturbing sensation was reported by the awake patient. Finally, the proximal part of the tined lead electrode was tunnelled to the upper part of the contralateral buttock, and connected to the percutaneous extension wire (model $3550-05$, Medtronic). This extension wire was again tunnelled to the contralateral buttock, where the wire was exteriorised and the screening cable (model 3550-03, Medtronic) was connected to the external pulse generator (model 3625, Medtronic). Permanent implantation took place immediately after the screening period if a reduction of symptoms of more than $50 \%$ was reported.

\section{Results}

We used video imaging to support percutaneous nerve evaluation and electrode implantation for sacral nerve stimulation, in 6 women with bowel disfunction. No intraoperative technical problem occurred. The median duration of screening period was 15 days (range, 7-22). During this period, we had no infection and no dislocation of the electrodes. All but one screening procedures were successful, and a permanent neurostimulator was implanted in five patients. Again, no complications, especially no wound infection at the lead or at the pocket of the stimulator in the gluteal area, were noted mean postoperative follow-up of 8 weeks.

\section{Discussion}

The minimally invasive video-assisted percutaneous nerve evaluation and the placement of the percutaneous tined lead were performed without any complications.

The use a camera to control the motor responses of the pelvic floor allows the potentially infected perianal area to be covered without narrowing the observer's view. Furthermore, with the use of an adequate optic and illumination, even small contractions of the pelvic floor are magnified (magnification, 3 to 4 times in our setting, depending on the optic and monitor) and better visualised than by simple eye observation. For surgeons, normally standing at the side of the patient's chest, it is more comfortable to watch the pelvic contraction on the monitor than the lean forward to look at the perianal area.

It is evident that operations in the perianal area have a high risk of infection, especially when artificial materials 


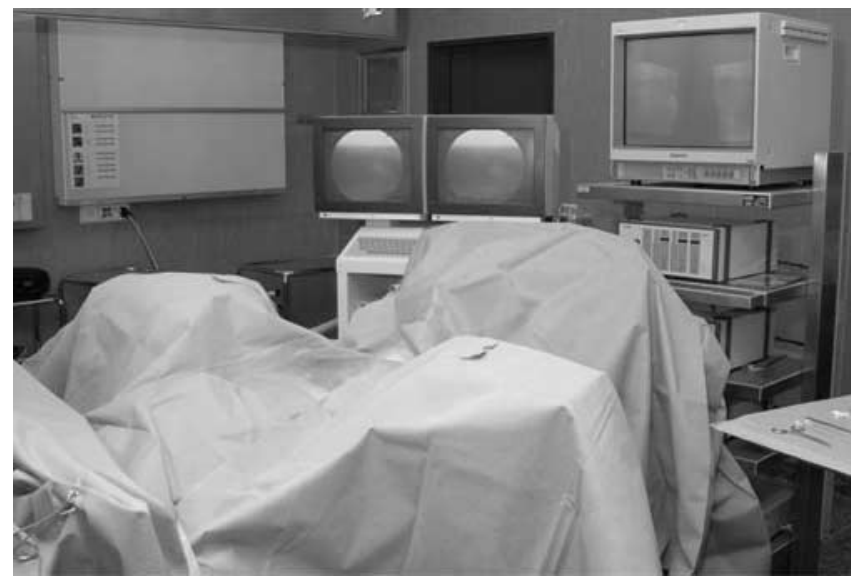

Fig. 2 After disinfections of the sacral area (cranially up to the iliac crest, laterally $15-20 \mathrm{~cm}$ from the anal verge on each side and caudally to the tip of the coccyx), sterile rectangular coverage is placed. The fluoroscope and the video optic, as well as the perianal area, are completely covered. The instrumental nurse will be on the left side of the patient's leg; the right-handed surgeon stays on the right side at the patient's thorax

are implanted. A lead infection close to the sacral roots is potentially a life-treating complication and therefore needs immediate surgical removal of the electrode, debridement of the wounds and treatment with antibiotics. All preventive measures to avoid direct contact to the perianal region should be undertaken. Therefore, some centres cover the perianal area with a transparent foil during the percutaneous nerve evaluation and the implantation of an electrode. These special foils are excellent barriers to bacteria or contaminated material. However, it is very difficult to attach these foils sufficiently around the anus. Additionally, steam often reduces the view through the transparent foil during the procedure. With the use of a camera, the area of consideration can be easily visualized and safely covered to avoid any contact with the perianal region. The two regions (anus and implantation site) are completely separated and contamination is very unlikely. The cover sheet is stacked at the level of the tip of the coccyx as shown in Fig. 2. The costs of the described camera are moderate as most clinics have endoscopic or laparoscopic equipment and therefore already have the adequate camera and monitor. Furthermore, the camera, the light cable and the support do not need to be sterilised or draped, which does not increase cost.

Our experience with no infection in six patients is promising. However, due to the small number of patients no statistically significant reduction in infection rate could be seen compared to the period before the use of video, where we had 4 infections in 40 procedures (10\%; unpublished data). Nevertheless, the use of the video during SNS is simple and does not increase costs, and the theoretical benefit is overwhelming. Thus, we have implemented the video-assisted technique as standard practice in our clinic.

\section{References}

1. Pescatori M, Meglio M, Cioni B, Colagrande C (1982) Colonic motility in two constipated neurological patients treated by spinal cord stimulation. In: Wienbeck M (ed) Motility of the digestive tract. Raven Press, New York, pp 541-547

2. Thanago EA, Schmidt RA, Orvis BR (1989) Neural stimulation for control of voiding dysfunction: a preliminary report in 22 patients with serious neuropathic voiding disorders. J Urol 142:340-346

3. Kenefick NJ, Christiansen J (2004) A review of sacral nerve stimulation for the treatment of faecal incontinence. Colorectal Dis 6:75-80

4. Jarrett ME, Mowatt G, Glazener CM et al (2004) Systematic review of sacral nerve stimulation for faecal incontinence and constipation. Br J Surg 91:1559-1569

5. Siegel SW, Catanzaro F, Dijkema HE et al (2000) Long-term results of a multicenter study on sacral nerve stimulation for treatment of urinary urge incontinence, urgency-frequency, and retention. Urology 56:87-91

6. Ripetti V, Caputo D, Ausania F et al (2002) Sacral nerve neuromodulation improves physical, psychological and social quality of life in patients with fecal incontinence. Tech Coloproctol 6:147-152

7. Spinelli M, Giardiello G, Gerber M et al (2003) New sacral neuromodulation lead for percutaneous implantation using local anesthesia: description and first experience. J Urol 170:1905-1907

8. Janknegt RA, Hassouna MM, Siegel SW et al (2001) Longterm effectiveness of sacral nerve stimulation for refractory urge incontinence. Eur Urol 39:101-106

9. Everaert K, Kerckhaert W, Caluwaerts H et al (2004) A prospective randomized trial comparing the 1-stage with the 2-stage implantation of a pulse generator in patients with pelvic floor dysfunction selected for sacral nerve stimulation. Eur Urol 45:649-654

10. Hetzer FH, Hahnloser D, Knoblauch Y et al (2005) New screening technique for sacral nerve stimulation under local anaesthesia. Tech Coloproctol 9:25-28

11. Rosen HR, Urbarz C, Holzer B et al (2001) Sacral nerve stimulation as a treatment for fecal incontinence. Gastroenterology 121:536-541

12. Uludag O, Koch SM, van Gemert WG et al (2004) Sacral neuromodulation in patients with fecal incontinence: a singlecenter study. Dis Colon Rectum 47:1350-1357

\section{Invited comment}

The work of Hetzer and colleagues concerns the "high" risk of sepsis $(20 \%)$ in patients submitted to sacral neuromodulation, suggesting that the use of videoscopy during installation is able to reduce the rate of sepsis. The authors report that the rate of sepsis during the procedure can reach up to $20 \%$ of patients. Nevertheless such a high rate was reported only once by Rosen et al. [1] and this 
not was confirmed by other series that reported infection rates of $2 \%-5 \%[2,3]$.

In my opinion, the results of Rosen et al. [1] should not be considered. Indeed, this series included a large number of patients with spinal injuries who were, therefore, potentially not responsive. This is the only work that emphasized a statistically significant increase of basal and squeezing pressures. Moreover, explantation occurred in 3 of 20 patients, 0 to 3 months after the procedure, while Hetzer and colleagues reported that the follow-up of the 6 patients was only 2 months. This cannot exclude that sepsis could occur later.

Hetzer and colleagues reported that during a 4-year experience before implementing videoscopy, only 1 explantation was necessary in a total of 36 procedures $(2.7 \%$ and not $3.4 \%)$. These data agree with those reported in the literature. However, this is not the $20 \%$ rate of infection that the authors stated in their introduction. In this paper, a total of 40 procedure with an infection rate of $10 \%$ (4 cases) was reported. How were these four cases treated? How many of these patients underwent explantation?
In conclusion, in my opinion, videoscopy is a nice idea, but the small number of patients in this study, the short follow-up and the lack of statistical conclusions do not fully validate the use of this technique.

V. Ripetti

Department of Surgery Campus Biomedico Rome, Italy

\section{References}

1. Rosen H, Urbaz C, Holzer B et al (2001) Sacral nerve stimulation as a treatment for faecal incontinence. Gastroenterology 121:536-537

2. Jarrett ME, Mowatt G, Glazener CM et al (2004) Systematic review of sacral nerve stimulation for faecal incontinence and constipation. Br J Surg 91:1559-1569

3. Spinelli M, Bertapelle P, Cappellano F et al (2001) Chronic sacral neuromodulation in patients with lower urinary tract symptoms: results from a national register. J Urol 166:541-545 